\title{
Calidad de vida relacionada con la salud en los servicios de Oncología del Hospital de Navarra. Grupo de Calidad de Vida de la EORTC
}

\author{
Health-related Quality of Life in the Oncology \\ departments of the Hospital of Navarra. \\ The EORTC Quality of Life Group
}

\author{
J. I. Arrarás ${ }^{1}$, F. Arias de la Vega ${ }^{2}$, J. J. Illarramendi ${ }^{3}$, A. Manterola ${ }^{2}$, E. Salgado ${ }^{3}$, \\ M. A. Domínguez ${ }^{2}$, R. Vera ${ }^{3}$
}

\begin{abstract}
RESUMEN
La evaluación de la calidad de vida es uno de los elementos clave de la atención que se ofrece al paciente oncológico. Este trabajo presenta la línea de investigación que sobre la calidad de vida se ha realizado desde 1992 en los servicios de oncología del Hospital de Navarra. Se trabaja dentro del Grupo de Calidad de Vida de la Organización Europea para la Investigación y Tratamiento del Cáncer -EORTC- en el desarrollo de cuestionarios y en otros proyectos. Se ha coordinado la creación de la escala de la EORTC de información. Se han validado instrumentos de medida de dicho organismo para nuestro país. Se han llevado a cabo estudios de calidad de vida en tumores y en satisfacción de los pacientes con los cuidados recibidos. Esta línea de investigación tiene un beneficio directo en la atención que reciben los pacientes.
\end{abstract}

Palabras clave. Calidad de Vida. EORTC. Cuestionario. Medición.

\begin{abstract}
Quality of life assessment is one of the key elements of the care that is offered to cancer patients. The aim of this work is to present the research line on quality of life that has been carried out since 1992 in the Oncology Departments of the Hospital de Navarra. These departments actively collaborate with the European Organisation of Research and Treatment of Cancer -EORTC- Quality of Life Group in creating questionnaires and also in other projects of this group. Our institution has coordinated the development process of the EORTC information module. Different EORTC questionnaires have been validated for use in our country. Quality of life studies have been carried out in the main tumour sites and in other areas, such as patients' satisfaction with care. This research line has a direct benefit on the attention that patients receive.
\end{abstract}

Key words. Quality of Life. EORTC. Questionnaire. Measurement.
1. Red de Salud Mental. Servicio Navarro de Salud. Pamplona.

2. Hospital de Navarra. Servicio de Oncología Radioterápica. Pamplona.

3. Hospital de Navarra. Servicio de Oncología Médica. Pamplona.

Recepción: 23 de junio de 2010

Aceptación provisional: 29 de octubre de 2010

Aceptación definitiva: 17 de diciembre de 2010

\section{Correspondencia \\ Juan Ignacio Arrarás, \\ Monasterio de Urdax 1-5ํㅡ D \\ 31007 Pamplona \\ jiarraras@correo.cop.es}

\section{Becas}

Este trabajo ha contado con la concesión de proyectos del Departamento de Salud del Gobierno de Navarra y de la Caja de Ahorros de Navarra. 


\section{INTRODUCCIÓN}

La calidad de vida (CV) de los pacientes y de las personas de su entorno es uno de los objetivos clave de los sistemas de salud. La evaluación de los tratamientos del cáncer tradicionalmente se había dirigido a medidas de tipo biomédico, como la respuesta al tumor, el intervalo libre de enfermedad o la supervivencia. A partir de los años 90 aumentó el interés por evaluar más formalmente el impacto de la enfermedad y los tratamientos en la CV. Dentro del campo de la oncología, la evaluación de la CV es actualmente una de las intervenciones más importantes que se realizan con el paciente oncológico y su entorno, intervención de gran utilidad para ayudar a ofrecer los mejores cuidados ${ }^{1}$.

Hace 20 años no existía prácticamente ningún instrumento de medición de $\mathrm{CV}$ creado y validado para ser contestado por los pacientes, y la mayoría de las pocas escalas disponibles se centraban únicamente en la evaluación por parte de los médicos de los aspectos físicos de la enfermedad, como el performance status ${ }^{2}$.

Con el presente artículo se pretende ofrecer una introducción al área de la valoración de la CV dentro de la oncología y aportar nuestra experiencia investigadora en este campo desde el año 1992, dentro del grupo de CV de la EORTC, grupo más importante en Europa de estudio de esta variable. Presentamos estudios de validación para nuestro país de los cuestionarios de la EORTC y estudios de evaluación de $\mathrm{CV}$ en pacientes tratados en los servicios de oncología del Hospital de Navarra.

\section{EVALUACIÓN DE CALIDAD DE VIDA DEL PACIENTE ONCOLÓGICO}

Existen diferentes definiciones del término calidad de vida. Aaronson ${ }^{3}$ plantea que la definición de la salud de la OMS como el bienestar físico, mental y social y no meramente la ausencia de enfermedad, es una base adecuada para definir la CV, y que en el campo de la oncología se deben incluir los efectos de la enfermedad y los tratamientos.

Actualmente, hay un acuerdo en las características de la CV que se consideran importantes, y que se tienen en cuenta a la hora de su medición: la subjetividad de la evaluación, la temporalidad, la multidimensionalidad y la relación con el estado de salud.

Debe ser el propio paciente quien mida su $\mathrm{CV}$, ya que se trata de un fenómeno subjetivo $^{3,4}$, donde los factores psicológicos pueden influir en la percepción del paciente sobre su enfermedad y su situación. Además, estas valoraciones de $\mathrm{CV}$ son temporales, y pueden variar debido a factores como los cambios que se producen en la situación del sujeto o en los criterios con los que éste evalúa dicha situación (el paciente puede aceptar que es normal que haya limitaciones en su vida). La CV es entendida como un concepto multidimensional ${ }^{5}$, cuya valoración debería incluir las diferentes áreas de la salud que se ven afectadas por la enfermedad y los tratamientos. La mayoría de los autores consideran las dimensiones de funcionamiento físico, social, psicológico, y las de los síntomas de la enfermedad y efectos secundarios de los tratamientos ${ }^{6,7}$.

La evaluación de la CV en oncología se lleva a cabo valorando diferentes áreas clave: protocolos de tratamientos (ensayos clínicos), los servicios de oncología de un hospital, un modelo para la atención de un grupo particular de pacientes (ej: los cuidados paliativos, el seguimiento de pacientes tras el alta) o la práctica clínica diaria ${ }^{4}$. En todas estas áreas, la CV es reconocida como una de las variables más importantes.

La mayoría de los ensayos clínicos incluyen una valoración de CV. Esta valoración se hace más importante, en casos en los que se estudian tratamientos para grupos de pacientes que se encuentran en estadios avanzados de la enfermedad, o cuando se quieren comparar tratamientos cuya efectividad en variables clínicas es cercana. En algunos países, como Canadá, la evaluación de CV es obligatoria en todo ensayo clínico. 
Para valorar la CV, es necesario poder contar con instrumentos de medida estandarizados, que tengan un buen funcionamiento psicométrico, donde la fiabilidad (consistencia interna, test-retest, armonía entre jueces), validez (criterio, constructoconvergente, divergente, de comparación entre grupos) y sensibilidad al cambio sean adecuadas. Estas mediciones de CV son el fruto de procesos de investigación, tanto en la creación de los instrumentos de evaluación, como de la metodología de aplicación y análisis.

\section{EL GRUPO DE CALIDAD DE VIDA DE LA EORTC}

La investigación en oncología se va dirigiendo progresivamente hacia una cooperación internacional más cercana ${ }^{1}$. La Organización Europea para la Investigación y Tratamiento del Cáncer (EORTC), es un organismo internacional en el que participan investigadores de diferentes países que trabajan en el área del cáncer.

La CV ha sido siempre un aspecto importante para la EORTC, tal como se desprende de los objetivos generales de esta organización: conducir, desarrollar, coordinar y estimular la investigación de laboratorio y clínica en Europa dirigida a incrementar el manejo del cáncer y de los problemas relacionados, a través de mejorar la supervivencia y también la calidad de vida ${ }^{2}$.

Dentro de este organismo hay un grupo de estudio sobre la CV en el que trabajan un espectro amplio de profesionales (oncólogos, psicólogos, estadísticos, sociólogos y otros) proveniente de Europa, EEUU y resto del mundo. Los objetivos generales de este grupo son el crear cuestionarios de medición de CV que puedan ser empleados en estudios internacionales; asesorar a los profesionales sobre el diseño, implementación y análisis de los estudios de CV; investigar sobre la metodología de la evaluación de la CV y la formación sobre la evaluación de $\mathrm{CV}^{2}$. Este equipo, dados sus componentes, experiencia y forma de trabajo, resulta muy adecuado para crear cuestionarios y desarrollar metodología de evaluación de CV.

\section{El sistema de medida de CV de la EORTC}

El sistema de medida de la CV de EORTC es un sistema modular formado por un cuestionario general, el cual recoge las áreas comunes del cáncer y sus tratamientos, que se complementa con módulos de contenido más específico. Este sistema permite comparar los resultados de diferentes trabajos y a la vez dar una respuesta a las preguntas concretas que puede plantear cada estudio ${ }^{8}$.

El cuestionario general de la EORTC QLQ-C30, en su versión tercera (EORTC QLQ-C30 versión 3.0) es el que está en la actualidad en uso ${ }^{1,9}$.

La EORTC define un módulo como un grupo de ítems que evalúan áreas que no se cubren, o no lo hacen suficientemente por el cuestionario general. Se han desarrollado diferentes módulos, como los de cáncer de pulmón EORTC QLQ-LC13 ${ }^{10}$, cáncer de mama EORTC QLQ-BR23 ${ }^{11}$, tumores de cabeza y cuello EORTC QLQ-H\&N35 ${ }^{9}$, cáncer colorrectal EORTC QLQ-CR38 ${ }^{12}$ y otros.

El sistema y la metodología de evaluación del grupo de CV de la EORTC, son utilizados por múltiples investigadores de dentro y fuera de este organismo, quienes además reciben formación y orientación ${ }^{13}$.

Los cuestionarios de la EORTC se emplean actualmente en: ensayos clínicos, estudios transversales o longitudinales de grupos de pacientes tratados en los servicios de oncología, y en la práctica clínica diaria. El QLQ-C30 es el cuestionario de CV más empleado en ensayos clínicos en Europa, y es también ampliamente utilizado en América y en el resto del mundo. Ha sido traducido y administrado en más de 80 idiomas $^{2}$.

\section{EXPERIENCIA EN EL HOSPITAL DE NAVARRA}

El Hospital de Navarra lleva participando desde 1992 en estudios del Grupo de CV de la EORTC dirigidos a la creación y validación de cuestionarios, y en asesoría a profesionales que realizan evaluaciones de 
$\mathrm{CV}$, siendo una de las instituciones que más colabora dentro de este grupo de investigación, y de toda la EORTC en general. Es el único centro español que ha sido miembro activo de este grupo de investigación en CV a lo largo de estos años. Este trabajo dentro de la EORTC nos ha permitido contar con instrumentos que recogen los aspectos de CV de los tumores más frecuentes y de otras áreas clave para el paciente oncológico. La incorporación a dicho grupo de investigación se dio a partir del interés en crear y contar con instrumentos de evaluación de CV, válidos a nivel internacional, que permitieran comparar resultados con los de otros países. Dichos instrumentos no estaban siendo creados ni adaptados para su uso en nuestro país.

\section{Estudios dentro del Grupo de calidad de vida de la EORTC}

Este grupo de investigación se ha reunido en Pamplona en dos ocasiones, en 1996 y 2009. Son las dos únicas veces en las que ha acudido a nuestro país.

A lo largo de estos años hemos trabajado en las distintas fases de creación y validación de cuestionarios de CV. En este sentido, hemos participado en estudios dirigidos al desarrollo de escalas como las de tumores de mama ${ }^{11}$, cabeza y cuello ${ }^{9}$, cáncer colorrectal ${ }^{14}$, satisfacción con los cuidados recibidos ${ }^{15} \mathrm{y}$ otros. Actualmente estamos involucrados en varios proyectos dirigidos a la creación y validación de escalas para diferentes tumores, tratamientos y dimensiones específicas. Gracias a estos proyectos hemos podido trabajar con una metodología de primera línea aplicable al desarrollo de escalas y a estudios multicéntricos.

Como único centro español participante en el Grupo de Calidad de Vida de la EORTC hemos aportado la visión de los pacientes y profesionales de nuestro país sobre qué aspectos son más importantes dentro de la $\mathrm{CV}$, cuál es nuestro funcionamiento en dimensiones clave como la sexualidad o la espiritualidad, y cómo puede ser más adecuado medir la CV. Además, hemos colaborado aportando datos al manual de valores de referencia, manual que ofrece a los investigadores las puntuaciones de CV esperables en el cuestionario general QLQC30 para determinados tumores y estadios de la enfermedad ${ }^{16}$.

\section{El módulo de información: EORTC QLQ-INFO25}

El cuestionario EORTC QLQ-INFO25, en el que hemos coordinado los diferentes pasos de su proceso de creación y validación -siendo la primera vez que un centro español coordina un estudio del Grupo de calidad de vida de la EORTC-, evalúa la información recibida por el paciente oncológico, el cual se puede encontrar en diferentes fases de la enfermedad y del proceso de tratamiento y seguimiento. Mide aspectos relacionados con la enfermedad, las pruebas diagnósticas, los tratamientos y otros cuidados que recibe el paciente. Además evalúa aspectos cualitativos sobre la utilidad y la satisfacción con esta información y el deseo de recibir más o menos información.

La información al paciente oncológico es uno de los elementos clave del soporte que reciben los pacientes por parte de los profesionales y cuidadores ${ }^{17}$. Está caracterizada por importantes diferencias culturales. Por ello, en la creación del cuestionario EORTC QLQ-INFO25 se ha pretendido dar especial atención a los aspectos de la información propios de las diferentes culturas de Europa y de otras zonas. Han participado en este estudio investigadores de 18 centros provenientes de las distintas áreas de Europa, Corea y Taiwán.

En la creación del EORTC QLQ-INFO25 se han seguido las Guías Oficiales del Grupo de calidad de vida de la EORTC para el desarrollo de instrumentos ${ }^{18}$. Las diferentes fases se han realizado a la vez en distintos países (Tabla 1) ${ }^{19-21}$ : 
Tabla 1. Fases en la creación del cuestionario de información EORTC QLQ-INFO25

Generación de una lista de áreas sobre la información al paciente oncológico: revisión de bases de datos de literatura científica y de otros cuestionarios

\begin{tabular}{l}
\hline Primera ronda de entrevistas semiestructuradas con profesionales y pacientes \\
\hline Construcción de un cuestionario provisional \\
\hline Segunda ronda de entrevistas semiestructuradas con profesionales y pacientes \\
\hline Cambios en la versión provisional del cuestionario \\
\hline Primer estudio multicéntrico: se entrevistan 133 pacientes \\
\hline Creación de una nueva versión provisional del cuestionario \\
\hline Estudio multicéntrico de validación, parte más amplia del estudio: 509 pacientes de diferentes áreas culturales \\
han rellenado el cuestionario de información EORTC QLQ-INFO25 en varias ocasiones \\
\hline
\end{tabular}

Este estudio de creación de la escala de información ha tenido, entre otras ventajas, las de coordinar una red amplia de investigadores, actuando además como impulsores del proyecto; profundizar en nuestra experiencia en la creación de instrumentos de CV; conocer mejor el campo de la información al paciente oncológico; y contar con un instrumento de medida que puede ser empleado a nivel internacional y que es útil para evaluar servicios, programas de mejora de la información, en la práctica clínica diaria y en otras áreas.

\section{Estudios de validación para España de los cuestionarios de calidad de vida de la EORTC}

En el grupo de CV de la EORTC se considera muy importante realizar estudios de validación del cuestionario general en cada país, contando además en cada estudio con una muestra de un tumor concreto. Las tres versiones del cuestionario general EORTC QLQ-C30 han sido traducidas y validadas para su uso en nuestro país con muestras de pacientes de cáncer de mama, pulmón, cabeza y cuello y próstata tratados en el Hospital de Navarra ${ }^{22,26}$. También se han validado para nuestro país los módulos de la EORTC para cáncer de pulmón ${ }^{27}$, mama ${ }^{28}$, cabeza y cuello ${ }^{29}$; cáncer colorrectal $^{30,31}$, próstata ${ }^{32}$ y de satisfacción con los cuidados recibidos ${ }^{33,34}$ que complementan al cuestionario general.

Según nuestra experiencia, para la validación de instrumentos, es clave intentar acoplar las mediciones de validación a las fechas en las que el paciente acuda al hospital y tenga una entrevista con los facultativos; contar con un tamaño de muestra que sea lo suficientemente amplio como para realizar adecuadamente los diferentes análisis psicométricos, aunque ello implique que el periodo de recogida de datos sea largo; intentar realizar varias mediciones con cada paciente a lo largo del periodo de tratamiento y seguimiento, que faciliten conocer la sensibilidad al cambio del cuestionario; evaluar otras variables clínicas y biográficas relacionadas con la situación del paciente (ej: edad, performance status...) que permitan conocer la capacidad del cuestionario para diferenciar grupos de pacientes organizados según estas variables; si es posible administrar una segunda escala de CV, de tal manera que podemos ver la relación de las puntuaciones entre áreas de ambos instrumentos cuyo contenido se supone que está más o menos alejado; realizar entrevistas que recojan la visión de los pacientes sobre el contenido y las expresiones de los ítems del cuestionario, ya que éste puede estar midiendo áreas donde haya diferencias culturales (ej: el papel de la familia), diferencias que es importante saber si son recogidas ade- 
cuadamente por el cuestionario, o sus expresiones puedan resultar molestas o confusas (ej: emplear o no la palabra cáncer).

\section{Estudios de calidad de vida en los servicios de Oncología del Hospital de Navarra}

Nuestro equipo lleva trabajando desde 1992 en estudios de evaluación de la CV de los pacientes oncológicos tratados en el Hospital de Navarra.

Estos estudios de CV se realizan en las principales localizaciones tumorales (mama, colorrectal...), y además en otras áreas, como la satisfacción con los cuidados recibidos. Las evaluaciones de CV se han incorporado como una variable clave al estudiar la efectividad de los tratamientos aplicados, y las características de un tumor. Hemos realizado varios estudios en cada localización tumoral, estudios que además han tendido a complementarse entre ellos, por lo que los podríamos considerar como constituyentes de líneas de investigación más específicas para cada tumor. Se ha buscado que estos estudios de CV ayuden a responder a preguntas clínicas importantes para los profesionales de los dos servicios de oncología, lo que ha incrementado la utilidad de dichos estudios.

$\mathrm{Al}$ principio se realizaban valoraciones de CV con muestras combinadas de pacientes en diferentes estadios de la enfermedad (local, avanzado) y que recibían también diferentes protocolos de tratamiento (ej: radioterapia combinada o no con quimioterapia). Estos estudios permitían comparar subgrupos de pacientes (ej: dentro del cáncer de mama, enfermedad localizada frente a avanzada), a la par que facilitaba la realización de los análisis de validación de los cuestionarios.

Posteriormente los estudios de CV se han dirigido a grupos con características más homogéneas (ej. pacientes en estadios iniciales de la enfermedad), y que recibían un (o uno de dos) protocolo (s) de tratamiento. Ello ha ayudado a conocer la CV de cada grupo de pacientes y a responder mejor a las preguntas de los profesionales.
Además, en varios de los estudios realizados hemos comparado el efecto diferencial en la CV de dos protocolos de tratamiento aplicables a pacientes con características clínicas similares. Esta información puede ser empleada tanto por los pacientes como los profesionales a la hora de elegir uno de los tratamientos: ej., puede darse el caso de que ambos tratamientos tengan el mismo efecto en variables clínicas como la supervivencia o la toxicidad, y diferente en la CV.

Inicialmente los estudios de CV tendían a contar con mediciones que se realizaban a lo largo del tratamiento y tras periodos de seguimientos cortos. Estas mediciones ayudaban a valorar, entre otros aspectos, la influencia de la toxicidad aguda de los tratamientos en la CV. Actualmente hay una demanda importante de llevar a cabo otras evaluaciones tras periodos de seguimiento más largos, lo que ayuda a conocer los efectos a corto plazo y a largo plazo de la enfermedad y los tratamientos. Esta información es útil, ya que es esperable que durante el periodo de seguimiento se den cambios en el estado clínico de los pacientes (principalmente mejoras) y, sobre todo, en los criterios a partir de los que valoran su situación (ej. los pacientes pueden tener una visión diferente del efecto de la cirugía en su CV cuando ha pasado un tiempo largo desde la intervención, el impacto de recibir el diagnóstico se ha atenuado y hay una mayor esperanza de que la enfermedad tenga una buena evolución, que cuando han recibido recientemente dicha intervención).

Se han llevado a cabo varios estudios con pacientes en estadios avanzados de la enfermedad, grupo clave para conocer su CV por la situación de la neoplasia, y la necesidad de hacer un balance entre la efectividad del tratamiento y su peso sobre la CV.

\section{Cáncer de mama}

En un primer trabajo, se midió la CV a lo largo del periodo de tratamiento y de seguimiento breve, en pacientes con cáncer de mama en diferentes estadios de la 
enfermedad (de local a metastático). Este estudio permitió comparar diferentes tipos de cirugía (radical, conservadora), tratamientos adyuvantes y diferentes estadios de la enfermedad ${ }^{35}$.

Los resultados más destacables fueron que las puntuaciones de CV de las pacientes eran en general altas. Valores más elevados de CV se encontraban en pacientes en estadios iniciales de la enfermedad. Las diferencias entre el tipo de cirugía en las pacientes en estadios iniciales de la enfermedad se daban a favor de la cirugía conservadora en aspectos emocionales (imagen corporal), aspectos que por otro lado son de gran importancia para la CV de las pacientes. Los tratamientos adyuvantes producían una afectación entre leve y moderada en aspectos específicos de la $\mathrm{CV}$, afectación que tendía a recuperarse en el periodo de seguimiento.

En un trabajo posterior se evaluó la $\mathrm{CV}$ en pacientes en estadios iniciales de la enfermedad, que se encontraban en un periodo de seguimiento de 5 años tras la finalización del tratamiento. Parte de esta muestra había sido incluida en el estudio anterior. Este estudio permitió además de conocer su CV, comparar sus valores en esta variable con los de la medición realizada al inicio del tratamiento, y evaluar las diferencias en CV entre tipos de cirugía tras una fase de seguimiento largo ${ }^{36}$.

Los valores de CV continuaban siendo elevados tras un periodo de seguimiento largo y las puntuaciones en toxicidad producida por los tratamientos eran muy bajas. Las diferencias entre el tipo de cirugía se seguían dando en aspectos emocionales. Ambos estudios reforzaban la administración de los tratamientos adyuvantes propuestos y orientaban hacia la aplicación de la cirugía conservadora en aquellas pacientes que deseaban y estaban en condiciones de optar por uno u otro tratamiento quirúrgico.

\section{Cáncer en pacientes de edad avanzada}

La evaluación y el cuidado de la CV de los pacientes oncológicos mayores son ob- jetivos prioritarios en la actualidad. En este sentido, hay una demanda importante de contar con datos de este sector de edad, entre otros motivos, debido a que hay un número creciente de pacientes mayores, los cuales además, están recibiendo con mayor frecuencia tratamientos oncológicos similares a los de las personas más jóvenes ${ }^{37}$.

Cáncer de mama: se ha evaluado a lo largo del periodo de tratamiento y seguimiento breves, a pacientes con enfermedad en estadios iniciales tratados con cirugía, radioterapia adyuvante y en algunos casos además hormonoterapia. Hemos adaptado el sistema de valoración de CV a las características del anciano, añadiendo una medida de actividades de la vida diaria. Este estudio nos ha permitido evaluar los diferentes tipos de cirugía (radical o conservadora) y también el efecto de la intervención en el ganglio centinela frente al vaciamiento ganglionar. En este trabajo se ha podido además comparar sus puntuaciones de CV con los valores aportados por pacientes más jóvenes ${ }^{38}$.

Las puntuaciones de CV de las pacientes han sido elevadas en la mayoría de las áreas evaluadas. Se han dado alteraciones leves en áreas relacionadas con la toxicidad de los tratamientos, que se recuperaban en el seguimiento. Las pacientes con cirugía conservadora y con intervención en el ganglio centinela presentaban mejor funcionamiento en dimensiones emocionales. Estas puntaciones eran muy cercanas a las ofrecidas por el grupo de mujeres más jóvenes, e indicaban que las pacientes entendían tolerar bien la enfermedad y los tratamientos oncológicos. Estos datos refuerzan la administración de este protocolo de tratamiento adyuvante en pacientes de más edad, y la aplicación de la cirugía conservadora y la del ganglio centinela, en los casos en que se cumplan los criterios para su administración.

\section{Cáncer de próstata}

Se ha evaluando la CV en pacientes en estadios iniciales de la enfermedad a lo largo del periodo tanto de tratamiento como 
de seguimiento largo (seis años), periodo del que existe una necesidad importante de contar con datos. Una parte de estos pacientes son mayores de 70 años, lo que nos ha permitido además profundizar en el estudio de la CV de este grupo de edad ${ }^{34}$. Estos pacientes recibían tratamiento de RT, acompañada o no de diferentes protocolos de hormonoterapia, dependiendo de las características del tumor.

Los valores de CV han sido en general elevados a lo largo de las diferentes mediciones. Se han dado puntuaciones algo más bajas en funcionamiento sexual. Se han producido afectaciones leves durante el periodo de tratamiento en dimensiones específicas de la toxicidad de la radioterapia, afectaciones que se han recuperado tras un periodo de seguimiento breve. Se ha producido una afectación progresiva en áreas de la toxicidad provocada por la hormonoterapia. Las diferencias entre grupos organizados por edad son leves, se han dado en pocas áreas (funcionamiento cognitivo, fatiga, síntomas urinarios). Estos datos apoyan el uso del tratamiento radioterápico combinado con hormonoterapia para este grupo de pacientes, incluyendo los pacientes de más edad.

\section{Tumores de cabeza y cuello}

Se ha realizado un número importante de estudios en esta localización tumoral. Dos de ellos, llevados a cabo con pacientes en estadios avanzados de la enfermedad, han permitido comparar dos protocolos de tratamiento de radio y quimioterapia que podían ser entendidos como intensivos. La CV se ha evaluado a lo largo de los períodos de tratamiento y seguimiento ${ }^{39}$.

Los resultados de estos estudios nos han indicado que los pacientes entendían su CV en general como moderadamente satisfactoria y toleraban los tratamientos combinados de radio y quimioterapia. Al administrar el tratamiento se observaban empeoramientos en áreas funcionales, psicosociales y de toxicidad. Estos datos apoyaban la administración de tratamientos combinados en estos pacientes. Además, se daban diferencias en áreas específicas de la CV entre los dos protocolos, lo que podía favorecer el entender uno de ellos como tratamiento de elección para pacientes con estas características clínicas.

Además, se ha evaluado la CV en pacientes con tumores de glotis, en estadios iniciales, que se encontraban en periodo de seguimiento (comunicación personal de los autores, congreso SEPO 2009). Se han comparado dos protocolos de tratamiento de radioterapia o cirugía.

Los valores de CV eran en general elevados. Alteraciones leves se han dado en áreas específicas de la $\mathrm{CV}$ relacionadas con la enfermedad y los tratamientos. Todo ellos indica que los tratamientos no habían dejado limitaciones importantes en los pacientes. Al comparar las dos modalidades de tratamiento, se han observado diferencias a favor de la radioterapia frente a la cirugía en voz y en funcionamiento emocional, lo que puede favorecer el uso del tratamiento radioterápico con estos pacientes.

\section{Cáncer colorrectal}

Se han llevado a cabo tres estudios en esta localización tumoral. En el primero se ha valorado la CV en pacientes con cáncer de colon o recto en enfermedad metastática, tratados con quimioterapia. Las evaluaciones de CV se han realizado a lo largo del tratamiento. Las puntuaciones en esta variable de los pacientes que han recibido el tratamiento eran en general moderadamente elevadas. Se han dado empeoramientos a lo largo de las mediciones en áreas relacionadas con la toxicidad y otros síntomas como la fatiga. Estas puntuaciones indicaban que el tratamiento se administraba a pacientes que podían tolerarlo adecuadamente y apoyaban el empleo de este protocolo de tratamiento, aspectos que son clave para los pacientes con enfermedad diseminada ${ }^{40}$.

Un segundo estudio se ha realizado con pacientes diagnosticados de cáncer de recto en enfermedad localmente avanzada tratados con quimio y radioterapia neoadyuvante. La CV se ha evaluado a lo largo del 
periodo de tratamiento y seguimiento. Las puntuaciones de CV han sido en general elevadas. Alteraciones leves y moderadas se han dado en las áreas de síntomas de la enfermedad, toxicidad de los tratamientos, fatiga, funcionamiento sexual, y en áreas funcionales tras la cirugía. Se han dado empeoramientos leves en toxicidad tras los tratamientos neoadyuvantes, y en áreas funcionales y fatiga tras la cirugía. Estos datos de $\mathrm{CV}$ indican que los pacientes han tolerado adecuadamente los tratamien$\operatorname{tos}^{41}$.

Se ha realizado un tercer estudio con pacientes diagnosticados de cáncer de recto estadios 2 o 3 , que se encontraban en periodo de seguimiento de al menos un año, tras recibir una de dos modalidades principales de cirugía (anastomosis-colostomía), radioterapia y quimioterapia neoadyuvante, y en la mayoría de los casos, quimioterapia adyuvante. Se han observado afectaciones entre leves y moderadas en áreas específicas de la CV relacionadas con la enfermedad y los tratamientos. Las diferencias entre los dos tratamientos quirúrgicos se han dado en una dimensión: una mayor frecuencia de deposiciones en los pacientes con conservación del esfínter. Esta información puede ser de utilidad para reforzar las intervenciones actuales, y a la hora de elegir por una modalidad de tratamiento ${ }^{31}$.

\section{Cáncer de pulmón}

Se ha llevado a cabo un estudio de CV a lo largo del tratamiento y un período de seguimiento breve, en pacientes con enfermedad no quirúrgica. En estos pacientes, tanto su enfermedad como los tratamientos de quimioterapia combinada o no con radioterapia pueden resultar agresivos para el paciente ${ }^{42}$.

Los pacientes que recibían los tratamientos presentaban una CV moderadamente satisfactoria. Se daban afectaciones de nivel medio en áreas relacionadas con la enfermedad y la toxicidad de los tratamientos. Estos datos apoyaban la administración de tratamientos activos y hacían ver que los pacientes que recibían tratamiento tenían un nivel autovalorado de CV que permitían pensar que podían tolerarlo.

\section{Satisfacción con los cuidados recibidos}

La satisfacción con los cuidados recibidos se ha evaluado en pacientes ambulatorios tratados en los servicios de Radioterapia Oncológica y de Oncología Médica. Ambos estudios nos permiten conocer que los aspectos de la atención ofrecida de habilidades técnicas, interpersonales y de administración de información reciben una valoración bastante elevada, y orientan hacia qué aspectos sería conveniente reforzar.

\section{ESTUDIOS ACTUALES}

En la actualidad, y como continuación de las investigaciones anteriores, estamos trabajando en diferentes estudios con pacientes mayores afectados de cáncer de mama y de pulmón.

Hemos trabajado con pacientes en estadios iniciales tratados con cirugía, radioterapia y en algunos casos hormonoterapia, en los que hemos buscado confirmar los resultados encontrados en la muestra inicial (presentados más arriba), incluyendo el efecto de la cirugía conservadora y del ganglio centinela, y conocer la CV de estas pacientes tras un periodo largo de seguimiento.

Además, hemos evaluado el efecto acumulado en la CV del tratamiento de hormonoterapia, en cáncer de mama en estadios iniciales (tratado además con cirugía combinada o no con radioterapia y/o quimioterapia). Se cuenta con pocos datos de este efecto acumulado, y menos, en población mayor. Se están realizando las mediciones de CV a lo largo de un año de administración de esta modalidad de tratamiento sistémico.

En los pacientes con cáncer de pulmón en estadios no quirúrgicos tratados con quimioterapia, buscamos profundizar en el conocimiento de la CV de este grupo de 
pacientes a lo largo del tratamiento y seguimiento, y conocer la influencia específica de la quimioterapia basada en el platino.

Un grupo de gran importancia clínica es el de las pacientes con cáncer de mama diagnosticadas en edad premenopáusica, dado el efecto que la enfermedad y los tratamientos pueden tener en aspectos clave, como el poder ser madre, la autoestima y otros. Estamos evaluando la CV en pacientes de este grupo que se encuentran en período de seguimiento.

También se están realizando otros estudios, como la evaluación del efecto en la $\mathrm{CV}$ de la radioterapia administrada con intención paliativa para tratar metástasis óseas. Este estudio puede ser de utilidad para conocer y en su caso intentar mejorar la efectividad de dicho tratamiento.

A lo largo de este tiempo hemos constatado que una gran mayoría de los pacientes colaboran en los estudios y valoran muy positivamente que se muestre interés en su CV. En este sentido, los pacientes agradecen además la posibilidad de contar con un profesional de la psicología, con el que poder comentar su visión de áreas clave de su CV.

\section{CONCLUSIONES Y DIRECTRICES FUTURAS}

Algunas de las áreas de trabajo que nos planteamos para el futuro serían las de: seguir desarrollando instrumentos de medida dentro de la EORTC que recojan aspectos clave de la CV, coordinando la creación de la escala de comunicación de este grupo de investigación. También vamos a trabajar con otras metodologías de creación de cuestionarios, como son las propias de la Teoría Item Respuesta ${ }^{4}$. Por otro lado, vamos a colaborar en la adaptación de los instrumentos de evaluación de la EORTC para su uso en otros países de habla hispana (aspecto del que existe actualmente una demanda importante). También pretendemos profundizar en el estudio de la CV dentro de cuestiones clínicas clave, como es la situación de los pacientes mayores o de aquellos en estadios avanzados de la enfermedad.
Agradecimientos

Nos gustaría agradecer a los diferentes profesionales de los servicios de Oncología Médica y Radioterápica su colaboración en los estudios de esta línea de investigación.

A Germán Jusué por su ayuda en la revisión del presente manuscrito.

\section{BIBLIOGRAFÍA}

1. Aaronson NK, Ahmedzai S, Bergman B, Bullinger M, Cull A, Duez M et al. The European Organization for Research and Treatment of Cancer QLQ-C30: a quality of life instrument for use in international clinical trials. J Nat Cancer Inst 1993; 85: 365-376.

2. Fayers P, Bottomley A. EORTC Quality of Life Group, Quality of Life Unit. Quality of life research within the EORTC - the EORTC QLQC30. European Organisation for Research and Treatment of Cancer. Eur J Cancer 2002; 38 Suppl 4: S125-133.

3. AARONSON NK. Assessment of quality of life benefits from adjuvant therapies in breast cancer. Recent Results Cancer Res 1993; 127: 201-210.

4. ОsовA D. Lessons learned from measuring health-related Quality of Life in oncology. J Clin Oncol 1994; 12: 608-616.

5. FAYERS P, MACHIN D. Quality of life: assessment, analysis and interpretation. Baffins Line: John Wiley and Sons, 2000.

6. Niezgoda HE, Pater JL. A validation study of the domains of the core EORTC quality of life questionnaire. Qual Life Res 1993; 2: 319-325.

7. Selby P. Measurement of Quality of Life in cancer patients. J Pharmacology 1993; 45: 384-386.

8. Aaronson NK, Cull A, KaAsa S, Sprangers M. The EORTC modular approach to quality of life assessment in Oncology. Int $\mathrm{J}$ Ment Health 1994; 23: 75-96.

9. BjoRdal K, DE GraefF A, Fayers PM, Hammerlid E, Van Pottelsberghe C, Curran D et al. A 12 country field study of the EORTC QLQ-C30 (version 3.0) and the head and neck cancer specific module (EORTC QLQ-H\&N35) in head and neck patients. Eur J Cancer 2000; 1; 36 (14): 1796-1807.

10. Bergman B, Aaronson NK, Ahmedzai S, KaAsa S, SulLIVAN M. The EORTC QLQ-LC13: a modular supplement to the EORTC core quality of life questionnaire (QLQ-C30) for use in lung 
cancer clinical trials. Eur J Cancer 1994; 30A: 635-642.

11. Sprangers M, Groenvold M, Arraras JI, Franklin $\mathrm{J}$, Velde A, Muller M et al. The EORTC Breast Cancer-Specific Quality-of-Life questionnaire Module (QLQ-BR23): first results from a three-country field study. J Clin Oncol 1996; 14 (10): 2756-2768.

12. Sprangers maG, Te Velde A, Aaronson NK. The construction and testing of the EORTC colorectal cancer specific Quality of Life questionnaire module (QLQ-CR38). Eur J Cancer 1999; 35: 238-247.

13. Вотtomley A, Aaronson NK. European Organisation for Research and Treatment of Cancer. International perspective on healthrelated quality-of-life research in cancer clinical trials: the European Organisation for Research and Treatment of Cancer experience. J Clin Oncol 2007; 25: 5082-5086.

14. Whistance RN, Conroy T, Chie W, Costantini A, SEZER O, Koller M et al. Clinical and psychometric validation of the EORTC QLQ-CR29 questionnaire module to assess health-related quality of life in patients with colorectal cancer. Eur J Cancer 2009; 45: 3017-3026.

15. Brédart A, Bottomley A, Blazeby JM, Conroy T, Coens C, D'Haese $S$ et al. An international prospective study of the EORTC cancer in-patient satisfaction with care measure (EORTC INPATSAT32). Eur J Cancer 2005; 41: 2120-2131.

16. Scott N, Fayers P, Aaronson N, Bottomley A, de GraefF A, Groenvold M et al. EORTC QLQ-C30. Reference values. Brussels: EORTC, 2008.

17. Rehnberg G, Absetz P, Aro AR. Women's satisfaction with information at breast biopsy in breast cancer screening. Patient Educ Couns 2001; 42: 1-8.

18. Blazeby J, Sprangers ma, Cull A, Groenvold M, Botтomley A. The EORTC Quality of Life Group. Guidelines for developing questionnaire modules, $3^{\text {rd }}$ Edition. Brussels: EORTC, 2002.

19. Arrarás JI, Kuljanic-Vlasic K, Bjordal K, Yun YH, EFFicace F, HolzNer B et al. EORTC QLQINFO26: a questionnaire to assess information given to cancer patients a preliminary analysis in eight countries. Psycho-Oncol 2007; 16: 249-254.

20. Arrarás Ji, Wright S, Greimel E, Holzner B, KulJanic-Vlasic K, Velikova G et al. Development of a questionnaire to evaluate the information needs of cancer patients: the EORTC questionnaire. Patient Educ Couns 2004; 54: 235241.
21. Arrarás Ji, Greimel E, Sezer O, Chie W, BergenMAR M, Costantini A et al. An international validation study of the EORTC QLQ-INFO25 questionnaire: an instrument to assess the information given to cancer patients. Eur J Cancer (En prensa).

22. ARRARÁs JI, ILlarRAMENDI JJ, VALERDI JJ. El cuestionario de Calidad de Vida para cáncer de la EORTC QLQ-C30. Estudio estadístico de validación con una muestra española. Rev Psic Salud 1993; 7: 13-33.

23. Arrarás Ji, Garrido E, Pruja E, Marcos M, TeJEDOR M, ARIAS F. El cuestionario de Calidad de Vida de la EORTC QLQ-C30 (versión 2.0). Estudio psicométrico con pacientes con cáncer de mama. Clínica Salud 2000; 11: 329-349.

24. Arrarás JI, Pruja E, Tejedor M, lllarramendi JJ, Domínguez MA, VALERDI JJ. El cuestionario de Calidad de Vida de la EORTC QLQ-C30 (versión 2.0). Estudio de validación para nuestro país con pacientes con cáncer de pulmón. Rev Oncología 1999; 1: 257-263.

25. Arrarás Ji, Arias F, Tejedor M, Pruja E, Marcos M, MARTínez E et al. The EORTC QLQ-C30 (version 3.0) quality of life questionnaire. Validation study for Spain with head and neck cancer patients. Psycho-Oncol 2002; 11: 249-256.

26. Arrarás Ji, Villafranca E, Arias de la Vega F, Domínguez MA, Lainez N, Manterola A et al. The EORTC quality of life questionnaire QLQ-C30 (version 3.0). Validation study for Spanish prostate cancer patients. Arch Esp Urol 2008; 61: 949-954.

27. Arrarás Ji, Pruja E, Marcos M, Tejedor M, IllaRRAMENDI JJ, VERA R et al. El cuestionario de Calidad de Vida para cáncer de pulmón de la EORTC QLQ-LC13. Estudio de validación para nuestro país. Oncología 2000; 23: 127134.

28. Arrarás JI, Tejedor M, lllarramendi JJ, Pruja E, Marcos M, ArIAS F et al. El cuestionario de calidad de vida para cáncer de mama de la EORTC, QLQ-BR23. Estudio psicométrico con una muestra española. Psic Conductual 2001; 9: 81-97.

29. Arrarás Ji, Arias F, Tejedor M, Vera R, Prujá E, Marcos $\mathrm{M}$ et al. El cuestionario de Calidad de Vida para tumores de cabeza y cuello de la EORTC QLQ-H\&N35. Estudio de validación para nuestro país. Oncología 2001; 24: 482491.

30. Arrarás Ji, Vera R, Manterota A, Martínez M, ARIAS F, Martínez E et al. El cuestionario de Calidad de Vida para cáncer colorrectal EORTC QLQ-CR38. Estudio de validación para nuestro país. Oncología 2003; 26: 285-292. 
31. Arrarás Ji, Suarez J, Arias de la Vega F, Vera R, Asin G, Arrazubi V et al. The EORTC Quality of Life Questionnaire for patients with colorectal cancer: EORTC QLQ-CR29 Validation study for Spanish patients. Clin Transl Oncol (En prensa).

32. Arrarás JI, Villafranca E, Arias de la Vega F, RoMERo P, Rico M, VILA M et al. The EORTC Quality of Life Questionnaire for patients with prostate cancer: EORTC QLQ-PR25. Validation study for Spanish patients. Clin Transl Oncol 2009; 11:160-164.

33. Arrarás JI, Vera R, Martínez M, Hernández B, Laínez $\mathrm{N}$, Rico $\mathrm{M}$ et al. The EORTC cancer in-patient satisfaction with care questionnaire: EORTC IN-PATSAT32. Validation study for Spanish patients. Clin Transl Oncol 2009; 11: 237-242.

34. Arrarás Ji, Rico M, Vila M, Chicata V, Asin G, MarTínez $M$ et al The EORTC cancer outpatient satisfaction with care questionnaire in ambulatory radiotherapy: EORTC OUTPATSAT35 RT. Validation study for Spanish patients. Psycho-oncol 2010; 19: 657-664.

35. Arrarás Ji, Illarramendi JJ, Tejedor M, Vera R, Pruja E, Marcos M et al. Quality of life in Spanish breast cancer patients assessed with the EORTC questionnaires. Rev Oncología 2000; 3: 100-106.

36. Arrarás JI, Illarramendi JJ, Manterola A, TEJedor M, VALERDI JJ, DomíngUEz MA. Evaluación de la calidad de vida a largo plazo en pacientes con cáncer de mama en estadios iniciales mediante cuestionarios de la EORTC. Rev Clin Esp 2003; 203: 577-581.
37. Repetto L, Comandini D. Cancer in the elderly: assessing patients for fitness. Crit Rev Oncol Haematol 2000; 35: 155-160.

38. Arrarás Ji, Manterola A, Domínguez MA, Arias F, VILLAFrAN-CA E, Romero P et al. Impact of radiotherapy on the quality of life of elderly patients with localized breast cancer. A prospective study. Clin Transl Oncol 2008; 10: 498-504.

39. Arias F, Domínguez MA, Arrarás JI, Villafranca E, Manterola A, Martinez E et al. Hyperfractionated radiation therapy and cisplatin for locally advanced head and neck cancer (LAHNC). A non-randomized comparison of Quality of Life between two consecutive treatment protocols at a single institution. Cancer therapy 2007; 5: 161-168.

40. Arrarás Ji, Vera R, Martínez M, Manterola A, Arias F, SALgado E. Quality of Life assessment through the EORTC questionnaires of colorectal cancer patients in advanced disease stages. Clin Transl Oncol 2006; 8: 664-671.

41. Arrarás Ji, Arias F, Vera R, Manterola A, Martínez M, Villafranca $\mathrm{E}$ et al. Quality of Life assessment through the EORTC questionnaires of locally advanced rectal cancer patients treated with preoperative chemoradiotherapy. Clin Transl Oncol 2006; 8: 423429.

42. Arrarás Ji, Martinez M, Manterola A, Salgado E, Martínez E, Vera R et al. Quality of life assessment in Spanish lung cancer patients by the EORTC questionnaires. Oncología 2005; 28: 174-182. 